\title{
ESTIMATIONS OF TOTAL MASS AND ENERGY OF THE OBSERVABLE UNIVERSE
}

\author{
Dimitar Valev \\ Department of Stara Zagora, Space Research and Technology Institute, \\ Bulgarian Academy of Sciences, 6000 Stara Zagora, Bulgaria
}

Received 2014-02-05; Revised 2014-03-18; Accepted 2014-03-21

\begin{abstract}
The recent astronomical observations indicate that the expanding universe is homogeneous, isotropic and asymptotically flat. The Euclidean geometry of the universe enables to determine the total gravitational and kinetic energy of the universe by Newtonian gravity in a flat space. By means of dimensional analysis, we have found the mass of the observable universe close to the Hoyle-Carvalho formula $M \sim c^{3} /(G H)$. This value is independent from the cosmological model and infers a size (radius) of the observable universe close to Hubble distance. It has been shown that almost the entire kinetic energy of the observable universe ensues from the cosmological expansion. Both, the total gravitational and kinetic energies of the observable universe have been determined in relation to an observer at an arbitrary location. The relativistic calculations for total kinetic energy have been made and the dark energy has been excluded from calculations. The total mechanical energy of the observable universe has been found close to zero, which is a remarkable result. This result supports the conjecture that the gravitational energy of the observable universe is approximately balanced with its kinetic energy of the expansion and favours a density of dark energy $\Omega_{\Lambda} \approx 0.78$.
\end{abstract}

Keywords: Flat Universe, Dimensional Analysis, Total Mass of the Universe, Total Energy of the Universe

\section{INTRODUCTION}

The problem for the average density of the universe $\bar{\rho}$ acquires significance when it has been shown that the General Relativity allows to reveal the geometry and evolution of the universe by simple cosmological models (Friedman, 1922; Lemaitre, 1927; Einstein and De Sitter, 1932). Crucial for the universe appears dimensionless total density $\Omega=\bar{\rho} / p_{c}$ where $\rho_{c}$ is the critical density of the universe. In the case of $\Omega<1$ (open universe) the global spatial curvature is negative and the geometry of the universe is hyperbolic and in the case of $\Omega>1$ (closed universe) the curvature is positive and the geometry is spherical. In the special case of $\Omega=1$ (flat universe) the curvature is zero and the geometry is Euclidean. Until recently scarce information has been available about the density and geometry of the universe. The most trustworthy total matter density $\Omega$ has been determined by measurements of the dependence of the anisotropy of the Cosmic Microwave Background (CMB) upon the angular scale. The recent results show that $\Omega \approx 1 \pm \Delta \Omega$, where the error $\Delta \Omega$ decreases from 0.10 (De Bernardis et al., 2000; Balbi et al., 2000) to 0.02 (Spergel et al., 2003), i.e., the density of the universe is close to the critical one and the universe is asymptotically flat (Euclidean).

The fact that $\Omega$ is so close to a unit is not accidental since only at $\Omega=1$ the geometry of the universe is flat and the flat universe was predicted from the inflationary theory (Guth, 1981). The total density $\Omega$ includes matter density $\Omega_{M}=\Omega_{b}+\Omega_{c}$, where $\Omega_{b} \approx 0.05$ is density of baryon matter and $\Omega_{c} \approx 0.22$ is density of cold dark matter (Peacock et al., 2001) and dark energy $\Omega_{\Lambda} \approx 0.73$ (Hinshaw et al., 2009) producing an accelerating expansion of the universe (Riess et al., 1998; Perlmutter et al., 1999). The found negligible CMB anisotropy $\frac{\delta T}{T} \sim 10^{-5}$ indicates that the early universe was very homogeneous and isotropic (Bennett et al., 1996). Three-dimensional maps of the distribution of galaxies corroborate homogeneous and 
isotropic universe on large scales greater than 100 Mps (Shectman et al., 1996; Stoughton et al., 2002).

Usually, Einstein pseudotensor is used for determination of the total energy of the universe (Rosen, 1994; Johri et al., 1995). This approach is general for open, close and flat anisotropic models, but pseudotensorial calculations are dangerous as they are very coordinate dependent and thus, they may lead to ambiguous results (Banerjee and Sen, 1997). Newtonian gravity still works reasonably in practically all gravitational problems, starting from earth gravity, space flights and star systems and ending to birth of stars and star clusters, with exception of extremely compact objects like black holes and neutron stars possessing strong gravitational field causing non negligible curvature of the space. After recent CMB observations discovered that the global geometry of the universe is flat, some cosmological problems could be solved by Newtonian gravity in Euclidean space. This opportunity has been used in the paper to estimate total mechanical energy of the observable universe.

To determine gravitational and kinetic energy of the observable universe, information of the size and total mass of the universe are needed. There are different estimations of the mass of the observable universe covering very large interval from $3 \times 10^{50} \mathrm{~kg}$ (Hopkins, 1980) to $1.6 \times 10^{60} \mathrm{~kg}$ (Nielsen, 1997). Also the estimations of the size (radius) of the universe are from 10 Glyr (Hilgevoord, 1994) to more than of 78 Glyr (Cornish et al., 2004).

\section{ESTIMATIONS OF THE TOTAL MASS AND SIZE (RADIUS) OF THE OBSERVABLE UNIVERSE}

Taking into account uncertainties of the estimations for the mass and size of the observable universe, an original approach for cosmology, namely dimensional analysis, has been applied below for estimation of the mass and size of the observable universe. The dimensional analysis is a conceptual tool often applied in physics to understand physical situations involving certain physical quantities. It is routinely used to check the plausibility of derived equations and computations. When the certain quantity, with which other determinative quantities would be connected, is known but the form of this connection is unknown, a dimensional equation was composed for its finding. Most often, the dimensional analysis is applied in mechanics where there are many problems having a few determinative quantities. The quantity of mass dimension in high energy physics is also obtained by means of the fundamental constants $c, G$ and $\hbar$. This is the famous Planck mass $m_{P l} \sim \sqrt{\hbar c / G} \sim 2.1710^{-8} \mathrm{~kg}$, whose energy equivalent-the Planck energy $E_{P l}=m_{P l} c^{2} \sim 10^{19} \mathrm{GeV}$ appears unification energy of the fundamental interactions.

The fundamental parameters as the gravitational constant $G$, speed of the light c and the Hubble constant $H \approx 70 \mathrm{~km} \mathrm{~s}^{-1} \mathrm{Mps}^{-1}$ (Mould et al., 2000) determine the global properties of the universe. Therefore, by means of these parameters, a mass dimension quantity $m_{x}$ related to the universe could be constructed:

$m_{x}=k c^{\alpha} G^{\beta} H^{\gamma}$

where, $\mathrm{k}$ is a dimensionless parameter of the order of magnitude of a unit and $\alpha, \beta$ and $\gamma$ are unknown exponents which have been found by means of analysis.

Taking into account the dimensions of the quantities in Equation 1 we obtain the system of linear equations for unknown exponents Equation 2:

$\alpha+3 \beta=0-\alpha-2 \beta-\gamma=0-\beta=1$

The determinant $\Delta$ of the system is Equation 3:

$\Delta=\left|\begin{array}{ccc}1 & 3 & 0 \\ -1 & -2 & -1 \\ 0 & -1 & 0\end{array}\right|=-1$

The determinant $\Delta \neq 0$, therefore the system has a unique solution. We find this solution by Kramer's formulae Equation 4:

$\alpha=\frac{\Delta_{1}}{\Delta}=3 \beta=\frac{\Delta_{2}}{\Delta}=-1 \quad \gamma=\frac{\Delta_{3}}{\Delta}=-1$

Thus, we find the mass $m_{x}$ related to the universe:

$m_{x} \sim \frac{c^{3}}{G H} \sim 10^{53} \mathrm{~kg}$

This value hits in the large interval for the mass of the universe mentioned at the end of Section 1 and coincides with Carvalho (1995) formula for the mass of the observable universe, deduced by totally different approach. Thus, the quantity $m_{x}$, obtained by means of analysis by means of the fundamental parameters c, G and $\mathrm{H}$, represents acceptable estimation of the mass of the observable 
universe. It is worthy to note that this value is independent from the cosmological model. First estimations of mass of the observable universe by means of dimensional analysis have been made from Valev (2009a).

The universe is flat and the total density, including dark matter and dark energy, is $\bar{\rho}=\Omega \rho_{\mathrm{c}} \approx \rho_{\mathrm{c}}$ where the critical density of the universe $\rho_{c}$ determines from Equation 6 (Peebles, 1971):

$\rho_{c}=\frac{3 H^{2}}{8 \pi G} \approx 9.510^{-27} \mathrm{~kg} \mathrm{~m}^{-3}$

Since the observable universe is homogeneous and isotropic, it appears 3-dimensional homogeneous sphere for an observer at arbitrary location. From Equation 5 and 6 we obtain:

$$
\bar{\rho}=\Omega \rho_{c}=\frac{3 H^{2} \Omega}{8 \pi G}=\frac{m_{x}}{V}=\frac{3 c^{3}}{4 \pi R^{3} G H}
$$

From Equation 7 we have estimated the size (radius) of the observable universe $R$ close to the Hubble distance $c H^{-1}$ :

$$
R=(2 / \Omega)^{1 / 3} \frac{c}{H} \sim c H^{-1}
$$

The result, obtained from Equation 8, shows the observable universe practically coincides with Hubble sphere. The Hubble sphere is the sphere where the recessional velocity of the galaxies is equal to the speed of the light in vacuum $\mathrm{c}$ and according to the Hubble law $v=c$ when $r=c H^{-1}$. Thus, the Hubble sphere appears a three-dimensional sphere, centered on the observer, having radius $r=c H^{-1}$ and density $\bar{\rho} \approx \rho_{c}$.

The mass of the observable universe would be deduced more precisely by means of the recent density of the universe $\bar{\rho}=\Omega \rho_{c}=\Omega \frac{3 H^{2}}{8 \pi G}$ and radius (size) of the observable universe $R \sim c H^{-1}$ :

$M=\frac{4}{3} \pi R^{3} \bar{\rho} \approx \frac{4}{3} \pi \frac{c^{3}}{H^{3}} \Omega \rho_{c}=\frac{c^{3}}{2 G H} \Omega$

This expression is more accurate than Equation 5, since the results of the dimensional analysis are correct with accuracy to a coefficient $k \sim 1$. This value practically coincides with the Fred Hoyle formula for the mass of the observable universe $M=\frac{c^{3}}{2 G H}$ (Kragh, 1999).
Any possible matter beyond the Hubble sphere recedes from the observer with superluminal velocity. Therefore, it does not affect the observer and it has no contribution in the mass and energy of the observable universe, calculated in relation to the observer.

Besides, we can estimate the total rest energy of the observable universe from Equation 9 and Einstein equation:

$$
E_{0}=M c^{2}=\frac{1}{2} \frac{c^{5}}{G H} \Omega \sim 10^{70} J
$$

\section{DETERMINATION OF THE TOTAL MECHANICAL ENERGY OF THE OBSERVABLE UNIVERSE}

The results of dimensional analysis and CMB observations suggest that the observable universe appears homogeneous 3-dimensional sphere with radius $\mathrm{R}$ close to Hubble distance $\mathrm{cH}^{-1}$. Hence, the gravitational potential energy $U$ of the observable universe is Equation 11:

$U=-G \int_{0}^{R} \frac{M(r) d m}{r}=-\frac{16}{3} G \pi^{2} \bar{\rho}^{2} \int_{0}^{R} r^{4} d r=-\frac{3}{5} \frac{G M^{2}}{R}$

where, 0 is an arbitrary location of the observer, $R \sim c H^{-1}$ is the radius of the observable universe and $M(r)=\frac{4}{3} \pi r^{3} \bar{\rho}$ is the mass of a sphere with radius $\mathrm{r}$.

According to the equivalence of mass and energy, dark energy also possesses mass and gravitational energy. Replacing Equation 8 and 9 in Equation 11 we have found the total gravitational energy of the observable universe:

$U=-\frac{3}{20} \frac{c^{5}}{G H} \Omega^{2}$

Similar approach has been used for calculation of the total gravitational energy of a body arising from gravitational interaction of the body with all masses of the universe (Woodward et al., 1975; Valev, 2009b).

Taking into account Equation 10 and 12 we find $U=-\frac{3}{10} \Omega M c^{2}$, i.e., the modulus of the total gravitational energy of the observable universe is close to $3 / 10$ of its total rest energy. 
The estimation of the total kinetic energy of the observable universe $\mathrm{T}$ is more complicated as a result of the diversity of movements of masses in the observable universe. We suggest that almost all kinetic energy of the observable universe is a result of the cosmological expansion since it includes movement of the enormous masses (galaxies and clusters of galaxies) with average speed of the order of magnitude of $c / 2$. The rotation curves of galaxies show that the majority of stars move into the galaxies with speed less than $v_{0}=3 \times 10^{5} \mathrm{~m} / \mathrm{s}$ (Sofue, 1996). Besides, on rare occasions, the peculiar (noncosmological) velocities of galaxies exceed this value (Strauss and Willick, 1995). On the other hand, the speed of medium-distanced galaxies (and their stars), as a result of the cosmological expansion is of the order of magnitude of $c / 2=1.5 \times 10^{8} \mathrm{~m} / \mathrm{s}$. Obviously, the kinetic energy of an "average star" in the observable universe, ensuing from its peculiar movement, constitutes less than $\left(2 v_{d} c\right)^{2} \sim 4 \times 10^{-6}$ part of its kinetic energy, ensuing from the cosmological expansion, therefore, former should be ignored.

Let us estimate the total kinetic energy of the observable universe in relation to an observer at arbitrary location. The total kinetic energy of the observable universe is the sum of the kinetic energy of all masses $m_{i}$ moving in relation to the observer with speed $v_{i}$ determined from Hubble (1929) law $v_{i}=H r_{i}$, where $r_{i} \leq$ $c H^{-1}$ is the distance between the observer and mass $m_{i}$ placed within the Hubble sphere. The linearity of the Hubble law has been precise confirmed in (Riess et al., 1998) by SNeIa observations. Newtonian formula $T=\frac{1}{2} \sum_{i} m_{i} v_{i}^{2}$ for kinetic energy was used in (Valev, 2009a), but the distant masses recede from the observer with speeds comparable with the speed of the light $c$. Therefore, the relativistic formula for kinetic energy is used below:

$$
T=c^{2} \sum_{i} m_{i}\left[\left(1-v_{i}^{2} / c^{2}\right)^{-1 / 2}-1\right]
$$

Since, for an arbitrary observer, the observable universe appears a 3-dimensional homogeneous sphere having radius $R \sim c H^{-1}$, Equation 13 can be replaced by the integral:

$$
\begin{aligned}
& T=c^{2} \int\left[\left(1-v^{2} / c^{2}\right)^{-1 / 2}-1\right] d m \\
& =4 \pi \bar{\rho} c^{2} \int_{0}^{R}\left[\left(1-v^{2} / c^{2}\right)^{-1 / 2}-1\right] r^{2} d r
\end{aligned}
$$

Replacing $v \approx v_{r}$ with expression from Hubble law $v_{r}$ $=H r$, Equation 14 transforms into:

$$
\begin{aligned}
& T=4 \pi \bar{\rho} c^{2} \int_{0}^{R}\left[\left(1-H^{2} r^{2} / c^{2}\right)^{-1 / 2}-1\right] r^{2} d r \\
& =4 \pi \bar{\rho} c^{2} I-\frac{4}{3} \pi \bar{\rho} c^{2} R^{3}
\end{aligned}
$$

Where:

$$
I=\int_{0}^{R}\left(1-H^{2} r^{2} / c^{2}\right)^{-1 / 2} r^{2} d r
$$

The solution of the integral $I$ is given from Equation 16:

$$
I=-\frac{c r}{2 H}\left(c^{2} / H^{2}-r^{2}\right)^{1 / 2}+\frac{c^{3}}{2 H^{3}} \arcsin \frac{H r}{c}
$$

Considering Equation 8 and replacing low and upper limits of integration we find:

$I=\frac{\pi}{4} \frac{c^{3}}{H^{3}}$

Replacing Equation 17 in Equation 15 we obtain:

$$
T=\pi^{2} \bar{\rho} \frac{c^{5}}{H^{3}}-\frac{4}{3} \pi \bar{\rho} \frac{c^{5}}{H^{3}}
$$

In consideration of $\bar{\rho}=\Omega \rho_{c}$ and Equation 6 we find the total kinetic energy of the observable universe:

$T=\frac{1}{2} \frac{c^{5}}{G H} \Omega\left(\frac{3 \pi}{4}-1\right)=M c^{2}\left(\frac{3 \pi}{4}-1\right)$

Taking into account Equation 12 and 19, the total mechanical energy of the observable universe determines from Equation 20:

$$
\begin{aligned}
& E=T+U=\frac{1}{2} \frac{c^{5}}{G H} \Omega\left(\frac{3 \pi}{4}-1-\frac{3}{10} \Omega\right)= \\
& M c^{2}\left(\frac{3 \pi}{4}-1-\frac{3}{10} \Omega\right) \approx 1.056 M c^{2} \sim M c^{2}
\end{aligned}
$$

Thus, the total mechanical energy of the observable universe is found close to its total rest energy. It is worthy to note that in the process of deducing the 
Equation 19 the dark energy was accepted as involved in cosmological expansion $\left(\Omega=\Omega_{M}+\Omega_{\Lambda}\right)$. But the dark energy has no kinetic energy; therefore it should be excluded from calculations of the total kinetic energy of the observable universe. In result, the density $\bar{\rho}=\Omega \rho_{\mathrm{c}}$ in Equation 18 must be replaced with $\rho_{M}=\Omega_{M} \rho_{c}$ and taking into account Equation 6 we find a relativistic Equation 21 for the total kinetic energy of the observable universe:

$$
T=\frac{1}{2} \frac{c^{5}}{G H} \Omega_{M}\left(\frac{3 \pi}{4}-1\right)=M c^{2} \Omega_{M}\left(\frac{3 \pi}{4}-1\right) \approx 1.356 \Omega_{M} M c^{2}
$$

The recent value of matter density is between $\Omega_{M}=0.19$ (Carlberg et al., 1997) and $\Omega_{M}=0.27$ (Hinshaw et al., 2009). As a result, the total kinetic energy of the observable universe $T \approx(0.26 \div 0.37) M c^{2}$, i.e., close to $3 / 10$ of its total rest energy $M c^{2}$.

Finally, from Equation 12 and 21, taking into consideration Equation 10 we find the total mechanical energy of the observable universe:

$$
\begin{aligned}
& E=T+U=\frac{1}{2} \frac{c^{5}}{G H}\left[\Omega_{M}\left(\frac{3 \pi}{4}-1\right)-\frac{3}{10} \Omega^{2}\right] \\
& =M c^{2}\left[\frac{\Omega_{M}}{\Omega}\left(\frac{3 \pi}{4}-1\right)-\frac{3}{10} \Omega\right] \\
& \approx(-0.04 \div 0.06) M c^{2} \approx(0 \pm 0.05) M c^{2}
\end{aligned}
$$

It is remarkably, that the total mechanical energy of the observable universe is close to zero. This result supports the conjecture that the gravitational energy of the universe is approximately balanced with its kinetic energy of the expansion (Lightman, 1984). According to Equation 22, the total kinetic energy of the observable universe is strictly equal to zero in case of $\Omega_{M}=\frac{3 \Omega^{2}}{10(3 \pi / 4-1)} \approx 0.22$. This value hits in the range of the recent estimations of matter density of the universe and it should be discussed as a prediction of the suggested model, which the future more accurate observations are able to test.

\section{CONCLUSION}

The recent astronomical observations indicate that the expanding universe is homogeneous, isotropic and asymptotically flat. The Euclidean geometry of the universe enables to determine the total kinetic and gravitational energies of the universe by Newtonian gravity in a flat space.
By means of an original approach for cosmology, namely dimensional analysis, a mass dimension quantity of the order of $10^{53} \mathrm{~kg}$, related to the universe, has been found close to Hoyle-Carvalho formula for the mass of the observable universe. This value is independent from the cosmological model and infers the size (radius) of the observable universe close to Hubble distance $\mathrm{cH}^{-1}$.

Both, the total kinetic and gravitational energies of the observable universe have been determined in relation to an observer at arbitrary location. Based on the simple homogeneous and isotropic model of the flat universe which expands according to Hubble law, we have found equation for the total gravitational energy of the observable universe. The modulus of the total gravitational energy of the observable universe is estimated to 3/10 of its total rest energy $M c^{2}$.

The relativistic calculations for total kinetic energy have been made and the dark energy has been excluded from examination. The total kinetic energy of the observable universe has been found close to the modulus of its total gravitational energy. Therefore, the total mechanical energy of the observable universe is close to zero, which is a remarkable result. This result supports the conjecture that the gravitational energy of the observable universe is approximately balanced with its kinetic energy of the expansion and favours a density of dark energy $\Omega_{\Lambda} \approx 0.78$.

\section{REFERENCES}

Balbi, A., P. Ade, J. Bock, J. Borrill and A. Boscaleri et al., 2000. Constraints on cosmological parameters from MAXIMA-1. Astrophys. J., 545: L1-L4. DOI: $10.1086 / 317323$

Banerjee, N. and S. Sen, 1997. Einstein pseudotensor and total energy of the universe. Pramana J. Phys., 49: 609-615. DOI: 10.1007/BF02848334

Bennett, C.L. A.J. Banday, K.M. Górski, G. Hinshaw and P. Jackson et al., 1996. Four-year COBE DMR cosmic microwave background observations: Maps and basic results. Astrophys. J., 464: L1-L4. DOI: $10.1086 / 310075$

Carlberg, R.G., H.K. Yee and E. Ellingson, 1997. The average mass and light profiles of galaxy clusters. Asrophys. J., 478: 462-475. DOI: 10.1086/303805

Carvalho, J.C., 1995. Derivation of the mass of the observable universe. Int. J. Theor. Phys., 34: 25072509. DOI: 10.1007/BF00670782

Cornish, N. J., D.N. Spergel, G.D. Starkman and E. Komatsu, 2004. Constraining the topology of the universe. Phys. Rev. Lett., 92: 201302-201305. DOI: 10.1103/PhysRevLett.92.201302 
De Bernardis, P., P.A.R. Ade, J.J. Bock, J.R. Bond and J. Borrill et al., 2000. A flat universe from highresolution maps of the cosmic microwave background radiation. Nature, 404: 955-959. DOI: $10.1038 / 35010035$

Einstein, A. and W. De Sitter, 1932. On the relation between the expansion and the mean density of the universe. Proc. Nat. Acad. Sci. USA., 18: 213-214. DOI: 10.1073/pnas.18.3.213

Friedman, A., 1922. Uber die krummung des raumes. Z. Physik, 10: 377-386. DOI: 10.1007/BF01332580

Guth, A.H., 1981. The inflationary universe: A possible solution to the horizon and flatness problems. Phys. Rev. D, 23: 347-356. DOI: 10.1103/PhysRevD.23.347

Hinshaw, G., J.L. Weiland, R.S. Hill, N. Odegard and D. Larson et al., 2009. Five-year Wilkinson Microwave Anisotropy Probe (WMAP1) observations: Data processing, sky maps and basic results. Astrophys. J. Suppl. Series, 180: 225-245. DOI: 10.1088/00670049/180/2/225

Hopkins, J., 1980. Glossary of Astronomy and Astrophysics. 1st Edn., Chicago Univ. Press, Chicago, ISBN-10: 9780226351711, pp. 224.

Kragh, H., 1999. Cosmology and Controversy. 1st Edn., Princeton University Press, Princeton, ISBN-10: 069100546X, pp: 488.

Hubble, E., 1929. A relation between distance and radial velocity among extra-galactic nebulae. Proc. Nat. Acad. Sci. USA., 15: 168-173. DOI: 10.1073/pnas.15.3.168

Johri, V.B., D. Kalligas, G.P. Singh and C.W. Everitt, 1995. Gravitational energy in the expanding universe. Gen. Relativ. Gravit., 27: 313-318. DOI: 10.1007/BF02109127

Lemaitre, G., 1927. Un univers homogène de masse constante et de rayon croissant rendant compte de la vitesse radiale des nébuleuses extra-galactiques. Ann. Soc. Sci. Brux., 47A: 49-59.

Lightman, A.P., 1984. Models of the early universe (Book Review). Science, 224: 863-863. DOI: 10.1126/science.224.4651.863

Mould, J.R., J.P. Huchra, W.L. Freedman, R.C. Kennicutt, Jr. and L. Ferrarese et al., 2000. The Hubble space telescope key project on the extragalactic distance scale. Combin. Constraints Hubble Constant. Astrophys. J., 529: 786-794. DOI: $10.1086 / 308304$

Nielsen, L., 1997. the extension, age and mass of the universe, calculated by means of atomic physical quantities and newton's gravitational constant.
Peacock, J.A., S. Cole, P. Norberg, C.M. Baugh and J. Bland-Hawthorn et al., 2001. A measurement of the cosmological mass density from clustering in the 2dF galaxy redshift survey. Nature, 410: 169-173. DOI: $10.1038 / 35065528$

Peebles, P.J., 1971. Physical Cosmology. 1st Edn., Princeton Univ. Press, Princeton, ISBN-10: 0691081085, pp: 296.

Perlmutter, S., G. Aldering, G. Goldhaber, R.A. Knop and P. Nugent et al., 1999. Measurements of omega and lambda from 42 high-redshift supernovae. Astrophys. J., 517: 565-586. DOI: 10.1086/307221

Hilgevoord, J., 1994. Physics and our View of the World. 1st Edn., Cambridge Univ. Press, Cambridge, ISBN-10: 9780521476805, pp: 316.

Riess, A.G., A.V. Filippenko, P. Challis, A. Clocchiatti and A. Diercks et al., 1998. Observational evidence from supernovae for an accelerating universe and a cosmological constant. Astron. J., 116: 1009-1038. DOI: $10.1086 / 300499$

Rosen, N., 1994. The energy of the universe. Gen. Relativ. Gravit., 26: 319-321. DOI: 10.1007/BF02108013

Shectman, S.A., D.S. Landy, A. Oemler, D.L. Tucker and H. Lin et al., 1996. The las campanas redshift survey. Astrophys. J., 470: 172-188. DOI: 10.1086/177858

Sofue, Y., 1996. The most completely sampled rotation curves for galaxies. Astrophys. J., 458: 120-131. DOI: $10.1086 / 176796$.

Spergel, D.N., L. Verde, H.V. Peiris, E. Komatsu and M.R. Nolta et al., 2003. First-year Wilkinson Microwave Anisotropy Probe (WMAP) observations: Determination of cosmological parameters. Astrophys. J. Suppl. Series, 148: 175-194. DOI: 10.1086/377226

Stoughton, C., R.H. Lupton, M. Bernardi, M.R. Blanton and S. Burles et al., 2002. Sloan digital sky survey: Early data release. Astron. J., 123: 485-548. DOI: $10.1086 / 324741$

Strauss, M.A. and J.A. Willick, 1995. The density and peculiar velocity fields of nearby galaxies. Phys. Reports, 261: 271-431. DOI: 10.1016/03701573(95)00013-7

Valev, D., 2009a. Determination of total mechanical energy of the universe within the framework of newtonian mechanics.

Valev, D., 2009b. Recent CMB observations enable to find the total gravitational energy of a mass.

Woodward, J.F., R.J. Crowley and W. Yourgrau, 1975. Mach's principle and the rest mass of the graviton. Phys. Rev. D, 11: 1371-1374. DOI: 10.1103/PhysRevD.11.1371 